\title{
Serum carcinoembryonic antigen and carbohydrate antigen 19-9 for prediction of malignancy and invasiveness in intraductal papillary mucinous neoplasms of the pancreas: A meta-analysis
}

\author{
WEILIN WANG ${ }^{1,2^{*}}$, LUFEI ZHANG ${ }^{1,2^{*}}$, LINGHUI CHEN $^{1,2}$, JIANFENG WEI $^{1,2}$, QIANG SUN $^{1,2}$, \\ QINGSHONG XIE ${ }^{1,2}$, XIAOHU ZHOU ${ }^{1,2}$, DONGKAI ZHOU ${ }^{1,2}$, PENGFEI HUANG ${ }^{1,2}$, \\ QIFAN YANG $^{1,2}$, HAIYANG XIE ${ }^{1}$, LIN ZHOU ${ }^{1}$ and SHUSEN ZHENG ${ }^{1,2}$
}

Key Laboratories of ${ }^{1}$ Combined Multi-Organ Transplantation, Ministry of Public Health, and ${ }^{2}$ Organ Transplantation,

The First Affiliated Hospital, College of Medicine, Zhejiang University, Hangzhou, Zhejiang 310003, P.R. China

Received January 31, 2014; Accepted September 16, 2014

DOI: 10.3892/br.2014.376

\begin{abstract}
The clinical importance of intraductal papillary mucinous neoplasms (IPMN) of the pancreas has been increasing due to the large number of newly diagnosed cases. A meta-analysis was used to assess the accuracy of serum carcinoembryonic antigen (CEA) and carbohydrate antigen 19-9 (CA19-9) for the identification of malignant and invasive IPMN. A literature search of PubMed and Web of Knowledge was conducted. Studies included in the analysis addressed the diagnostic accuracy of serum CEA and CA19-9 and pooled estimates of sensitivity, specificity, positive- and negative-likelihood ratios (PLR and NLR), diagnostic odds ratio (DOR) and receiver operating characteristic curves were calculated using random-effects models. Predefined subgroup analysis was performed. Fifteen studies (published between 2001 and 2013) were analyzed, including a total of 1,629 patients. Pooled estimates of CEA in malignant and
\end{abstract}

Correspondence to: Professor Shusen Zheng, Key Laboratory of Combined Multi-Organ Transplantation, Ministry of Public Health, The First Affiliated Hospital, College of Medicine, Zhejiang University, 79 Qingchun Road, Hangzhou, Zhejiang 310003, P.R. China

E-mail: shusenzheng@zju.edu.cn

${ }^{*}$ Contributed equally

Abbreviations: CI, confidence interval; DOR, diagnostic odds ratio; LR, likelihood ratio; NLR, negative-likelihood ratio; PLR, positive-likelihood ratio; QUADAS, quality assessment of diagnostic accuracy studies; FN, false-negative; IPMN, intraductal papillary mucinous neoplasms; CEA, carcinoembryonic antigen; CA19-9, carbohydrate antigen 19-9; CIS, carcinoma in situ

Key words: sensitivity, specificity, carcinoembryonic antigen, carbohydrate antigen 19-9, intraductal papillary mucinous neoplasms, positive-likelihood ratio, negative-likelihood ratio, diagnostic odds ratio invasive IPNM prediction were: Pooled sensitivity, 18 and 18\%; pooled specificity, 93 and 95\%; PLR, 2.83 and 3.54; NLR, 0.89 and 0.89; and DOR, 3.35 and 3.6, respectively. Pooled estimates of CA19-9 in malignant and invasive IPMN prediction were: Pooled sensitivity, 40 and 52\%; pooled specificity, 89 and 88\%; PLR, 2.93 and 3.78; NLR, 0.74 and 0.6; and DOR, 4.34 and 6.33, respectively. In conclusion, serum CEA has low sensitivity and high specificity for malignant and invasive IPMN. Serum CA19-9 is a useful non-invasive preoperative tool for differentiating between invasive and benign IPMN and should be taken into account in the decision to perform surgery.

\section{Introduction}

Intraductal papillary mucinous neoplasms (IPMN) of the pancreas is a rare pancreatic disease. Since the World Health Organization (WHO) defined intraductal papillary mucinous tumor (1) in 1996 [renamed IPMN in 2000 (2)], detection and awareness have increased. Classification of IPMN as according to the WHO nomenclature is as follows: Adenoma, borderline tumor, carcinoma in situ (CIS) and invasive carcinoma (1). However, the classification of IPMN according to the 2010 WHO criteria is as follows: Low-, intermediate-, high-grade dysplasia and invasive carcinoma. IPMN was classified into three subtypes: Branch, main and mixed duct-type $(3,4)$. Furthermore, much remains unknown regarding the incidence of malignancy, postoperative prognosis and natural course of this disease, despite the increasing number of patients diagnosed with IPMN. An IPMN has malignant potential; it first transforms from an adenoma to a borderline neoplasm followed by carcinoma, ultimately becoming invasive $(5,6)$. Tumor resection is the only curative treatment for malignant and invasive IPMN. Benign and asymptomatic IPMN may be monitored and do not require surgical treatment (7-9). Accurate assessment of the likelihood of malignancy or invasion is required for appropriate management of IPMN.

Various modalities, including computerized tomography, endoscopic ultrasound and magnetic resonance, have been 
advocated for differentiation of benign from malignant IPMN. However, accurate diagnosis and preoperative assessment are crucial (10), even with modern cross-sectional imaging it remains difficult to predict malignancy correctly (11). Analysis of cystic fluid from fine-needle aspiration has also been used to distinguish malignant tumors and potentially pre-malignant mucinous cystic neoplasms from pseudocysts and serous cystadenomas (12). Cyst fluid carcinoembryonic antigen (CEA) and carbohydrate antigen 19-9 (CA19-9) are the most accurate tests available for the identification of malignant cystic lesions of the pancreas (13), but CEA and CA19-9 are of limited value in differentiating malignant from benign disease (14). In patients with resectable ductal adenocarcinoma, raised serum levels of CA19-9 and CEA have been shown to predict the stage and survival rate (15-17). The role of serum CEA and CA19-9 in predicting malignant and invasive IPMN remains controversial. The aim of the present meta-analysis was to determine the diagnostic precision of serum CEA and CA19-9 in predicting malignant and invasive IPMN.

\section{Patients and methods}

Selection of studies and subgroup categories. A comprehensive literature search of PubMed and Web of Knowledge using the terms '(IPMN OR Intraductal papillary mucinous neoplasm) AND Pancrea*' was performed. Two investigators (Lufei Zhang and Linghui Chen) independently reviewed each study for fulfillment of the predefined inclusion criteria.

The inclusion criteria were as follows: i) English language; ii) full-manuscript publication; iii) publication year, 2001-2013; iv) study design (prospective cohort, retrospective cohort and case series); v) study population (patients with IPMN confirmed by histology); and vi) minimum score of $11 / 14$ in the quality assessment of diagnostic accuracy studies (QUADAS) checklist. Criteria for exclusion were: i) A specific serum CEA or CA19-9 cut-off value was not used to evaluate the sensitivity and specificity of differentiation of benign from malignant IPMN; ii) insufficient information to construct $2 \times 2$ contingency tables; and iii) editorials, review studies, duplicate publications and case reports.

Thresholds of $5 \mathrm{ng} / \mathrm{ml} \mathrm{CEA}$ and $35 \mathrm{ng} / \mathrm{ml} \mathrm{CA19-9}$ have been used in published studies. Studies that included these thresholds were included in the meta-analysis despite serum CEA and CA19-9 being continuous variables.

Subgroup analysis. The following information was used to perform subgroup analysis: Year of publication, study location, number of patients, number of centers involved, study design and QUADAS scores. The effects of these variables on the sensitivity and specificity or inconsistency of CEA and CA19-9 for the diagnosis of malignant and invasive IPMN were assessed.

Statistical analysis. Outcomes of interest for diagnosis of malignant invasive in IPMN included: Sensitivity, specificity, likelihood ratios and area under the summary receiver operating characteristic curves (sROC) of CEA and CA19-9. Malignant IPMN was defined as CIS or invasive carcinoma. Data were extracted in the form of the true-positive, true-negative, false-negative (FN) and false-positive to construct $2 \times 2$ tables.

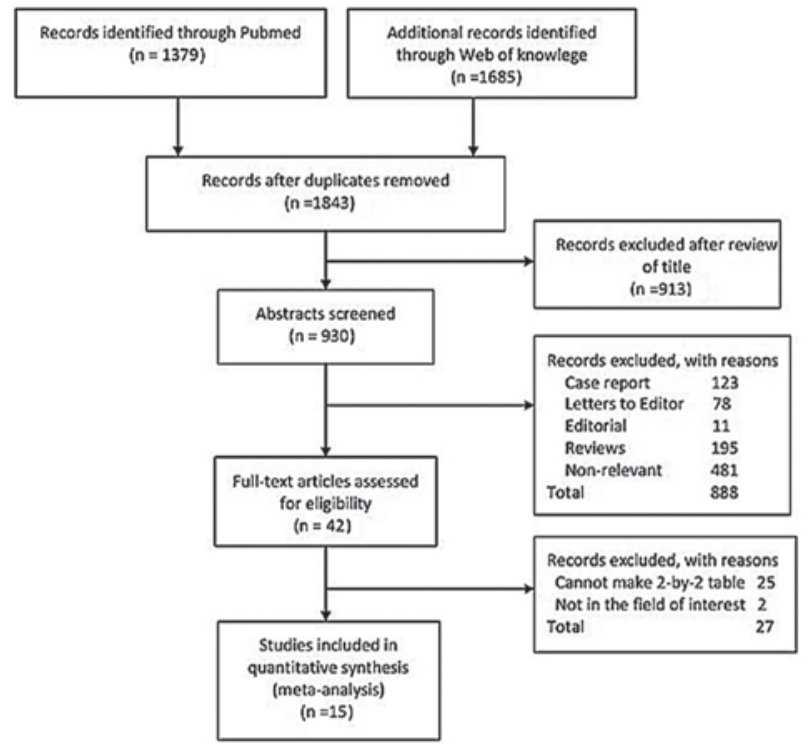

Figure 1. Flow chart demonstrating the algorithm for identifying suitable studies for inclusion.

Data were analyzed using the statistical software package Meta-DiSc, version 1.4 (Clinical Biostatistics Unit, Ramón and Cajal Hospital, Madrid, Spain) (18). Pooled estimates of sensitivity, specificity, positive-likelihood ratio (PLR), negative-likelihood ratio (NLR), diagnostic odds ratio (DOR) [with a 95\% confidence interval (CI)] were calculated using the random-effects model. sROC was used to assess the interaction between sensitivity and specificity. DOR and area under the sROC curve (AUC) were used to analyze the diagnostic performance of serum CEA, and CA19-9 was used to distinguish benign from malignant IPMN and to differentiate non-invasive from invasive IPMN. $\mathrm{I}^{2}$ expresses the variation across studies according to heterogeneity in the form of a percentage. The $\mathrm{I}^{2}$ value is $0-100 \%$, with $0 \%$ being a completely homogeneous study. A random-effects pooling method was used for high $\mathrm{I}^{2}(>50 \%)$. Threshold analysis was performed using the Spearman coefficient to identify the correlations between sensitivity and specificity. ROC curves are the optimum summary measure of performance in place of pooled indices when a threshold effect is present.

Heterogeneity was explored by means of a subgroup analysis. Studies were allocated to pre-specified subgroups according to location, number of centers, QUADAS score and type of IPMN. Meta-regression using variables in the subgroup-analysis as 'dummy' variables for each category was performed to determine the main sources of heterogeneity.

\section{Results}

Systematic review. Of the 1,843 studies, 1,801 were excluded following a preliminary abstract review, leaving 42 for detailed full-text evaluation. A total of 15 met the inclusion criteria $(9,19-32)$. The number of studies included is outlined in Fig. 1, as according to the reason for exclusion at each stage of assessment. The included studies involved 1,530 patients; the characteristics of the included studies are shown in Table I. 
A

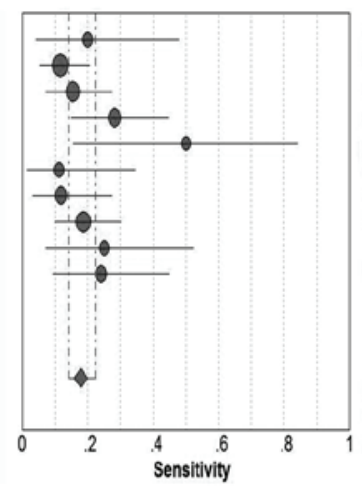

C

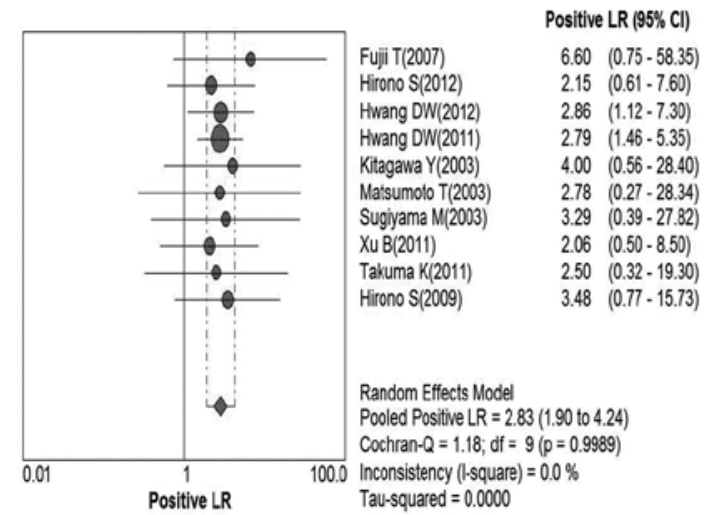

Pooled Sensitivity $=0.18$ (0.14 to 0.22$)$ Chi-square $=12.13 ; d f=9(p=0.2061)$ inconsistency (1-square) $=25.8 \%$

\section{(D)}

Positive LR $195 \% \mathrm{C}$

$6.60(0.75-58.35)$

$2.15(0.61-7.60)$

$2.79(1.46-5.35)$

$4.00 \quad(0.56-28.40)$

$2.78 \quad(0.27-28.34$

$(0.32-19.30)$

$(0.77-15.73)$
B

0

D

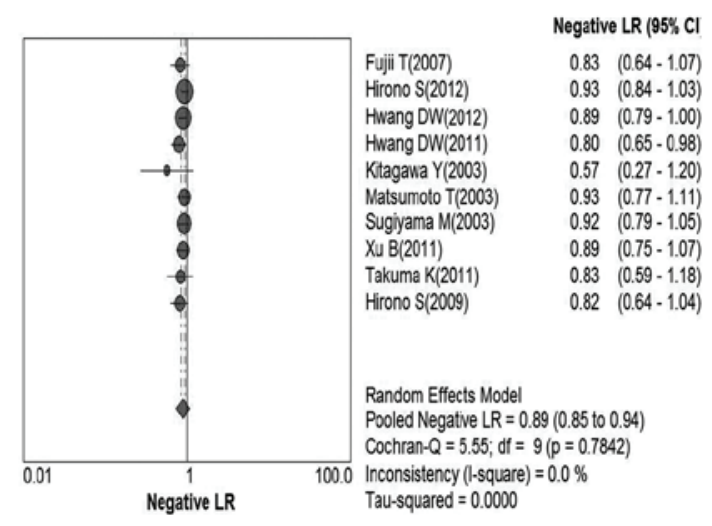

Specificity ( $95 \% \mathrm{Cl})$

$0.97(0.84-1.00)$

$0.95(0.85-0.99)$

$0.95(0.89-0.98)$

$0.90(0.85-0.94)$

$0.88(0.47-1.00)$

$0.96(0.80-1.00)$

$0.96(0.82-1.00)$

$0.91(0.71 \cdot 0.99)$

$0.90(0.55-100)$

$0.93(0.77-0.99)$

Figure 2. Forest plots of serum carcinoembryonic antigen for predicting malignant intraductal papillary mucinous neoplasms. (A) Sensitivity; (B) specificity; (C) positive-likelihood ratio (LR); and (D) negative-LR. CI, confidence interval.

\section{Meta-analysis}

Serum CEA for malignant IPMN. In total, 10 studies were included for analysis (9,20-24,29-32). Pooled sensitivity was $18 \%$ (95\% CI, 14-22\%), pooled specificity was $93 \%$ (95\% CI, 90-95\%), PLR was 2.83 (95\% CI, 1.90-4.24), NLR was 0.89 (95\% CI, 0.85-0.94), DOR was 3.35 (95\% CI, 2.09-5.36) and $\mathrm{I}^{2}=0.0 \%$. Parameters were homogenous and there was a significant correlation between sensitivity and specificity, indicating a threshold effect; therefore, a random-effects method was used. Forest plots of all indices of diagnostic accuracy are shown in Fig. 2. Results were plotted as a symmetrical sROC curve (Fig. 3). The AUC was 0.69 [standard error (SE), 0.03].

Serum CEA for invasive IPMN. A total of 9 studies were included $(9,20,22,24,25,28-30,32)$. Pooled sensitivity was $18 \%$ (95\% CI, 13-23\%), pooled specificity was $95 \%$ (95\% CI, 93-96\%), PLR was 3.54 (95\% CI, 2.17-5.77), NLR was 0.89 (95\% CI, 0.84-0.94), DOR was 3.6 (95\% CI, 2.14-6.06) and $\mathrm{I}^{2}=0.0 \%$. Parameters were homogenous and there was a significant correlation between sensitivity and specificity, indicating a threshold effect; therefore, a random-effects method was used. Forest plots of all the indices of diagnostic accuracy are shown in Fig. 4. Results were plotted as a symmetrical sROC curve (Fig. 5). The AUC was 0.70 (SE, 0.03).

Serum CA19-9 for malignant IPMN. In total, 15 studies were included $(9,19-32)$. Pooled sensitivity was $40 \%$ (95\% CI, 36-44\%), specificity was 89\% (95\% CI, 87-91\%), PLR was 2.93 (95\% CI, 2.10-4.09), NLR was 0.74 (95\% CI, 0.64-0.84), DOR was 4.34 (95\% CI, 2.65-7.10) and $\mathrm{I}^{2}=58.6 \%$. These parameters were highly heterogeneous; therefore, a random-effects

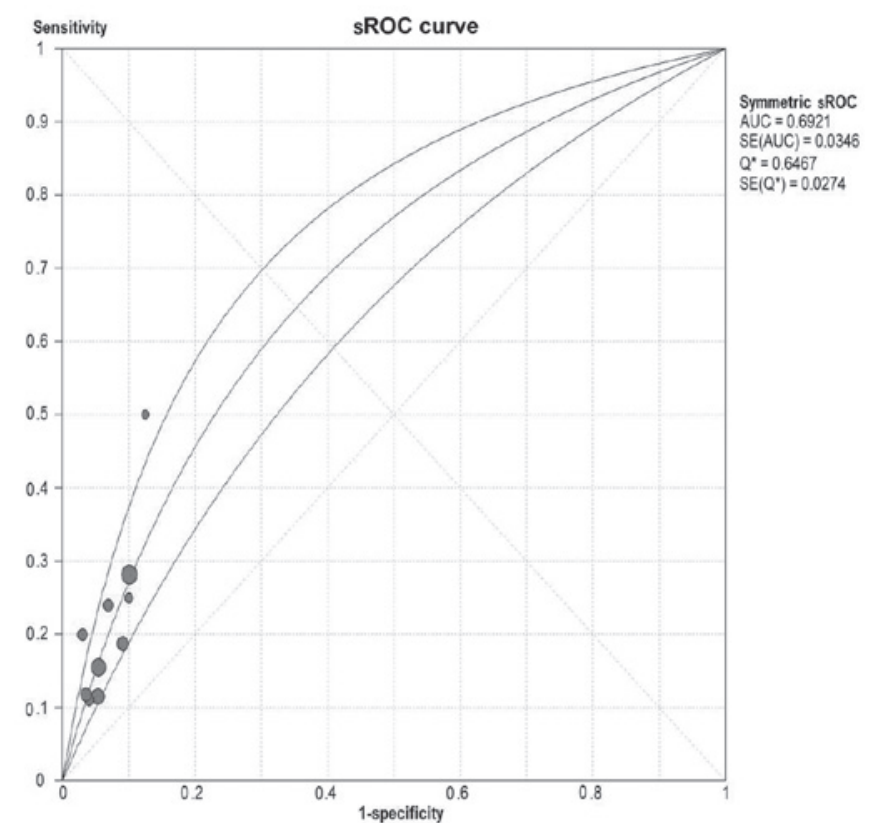

Figure 3. sROC curve of serum carcinoembryonic antigen for predicting malignant intraductal papillary mucinous neoplasms. sROC, summary receiver operating characteristic; AUC, area under the sROC curve; SE, standard error.

method was used. Fig. 6 shows forest plots of all the indices of diagnostic accuracy with heterogeneity denoted. Results were plotted as a symmetrical sROC curve (Fig. 7) and the AUC was 0.73 (SE, 0.03). There was no significant correlation between 
Table I. Characteristics of the included studies evaluating the performance of serum CEA and CA19-9 for predicting malignant and invasive IPMN.

\begin{tabular}{|c|c|c|c|c|c|c|c|c|c|c|}
\hline Author & Year & Country & Centres, $\mathrm{n}$ & Design & Patients, $\mathrm{n}$ & $\begin{array}{c}\text { Mean age, } \\
\text { years }\end{array}$ & Male, $\mathrm{n}$ & $\begin{array}{l}\text { QUADAS } \\
\text { scores }\end{array}$ & $\begin{array}{l}\text { Type of } \\
\text { IPMN }\end{array}$ & (Refs.) \\
\hline Fujii et al & 2007 & Japan & 1 & Retrospective & 51 & NA & NA & 11 & $\mathrm{All}^{\mathrm{a}}$ & (30) \\
\hline Hirono et al & 2012 & Japan & 1 & Retrospective & 144 & NA & 74 & 12 & Branch $^{\mathrm{b}}$ & (21) \\
\hline Hwang et al & 2012 & South Korea & 1 & Retrospective & 187 & 63.4 & 114 & 13 & $\mathrm{All}^{\mathrm{a}}$ & (20) \\
\hline Sadakari et al & 2010 & Japan & 1 & Retrospective & 53 & NA & NA & 11 & Branch $^{\mathrm{b}}$ & (26) \\
\hline Hwang et al & 2011 & South Korea & 11 & Retrospective & 237 & 63.1 & 137 & 13 & Branch $^{\mathrm{b}}$ & (24) \\
\hline Ohtsuka et al & 2012 & Japan & 1 & Retrospective & 99 & NA & 60 & 12 & Branch $^{\mathrm{b}}$ & (19) \\
\hline Kitagawa et al & 2003 & USA & 1 & Retrospective & 42 & NA & NA & 11 & $\mathrm{All}^{\mathrm{a}}$ & (32) \\
\hline Matsumoto et al & 2003 & Japan & 1 & Retrospective & 43 & NA & 32 & 12 & Branch $^{\mathrm{b}}$ & (31) \\
\hline Lee et al & 2010 & South Korea & 1 & Retrospective & 119 & NA & NA & 11 & $\mathrm{All}^{\mathrm{a}}$ & (27) \\
\hline Sugiyama et al & 2003 & Japan & 1 & Retrospective & 51 & NA & 39 & 12 & $\mathrm{All}^{\mathrm{a}}$ & (9) \\
\hline $\mathrm{Xu}$ et al & 2011 & China & 1 & Retrospective & 86 & NA & 62 & 12 & $\mathrm{All}^{\mathrm{a}}$ & (22) \\
\hline Takuma et al & 2011 & Japan & 1 & Retrospective & 46 & NA & 29 & 12 & Main $^{c}$ & (23) \\
\hline Nara et al & 2009 & Japan & 1 & Retrospective & 123 & 64.7 & 70 & 13 & $\mathrm{All}^{\mathrm{a}}$ & (28) \\
\hline Hirono et al & 2009 & Japan & 1 & Retrospective & 54 & NA & 31 & 12 & $\mathrm{All}^{\mathrm{a}}$ & (29) \\
\hline Shin et al & 2010 & Korea & 1 & Retrospective & 195 & NA & NA & 11 & $\mathrm{All}^{\mathrm{a}}$ & (25) \\
\hline
\end{tabular}

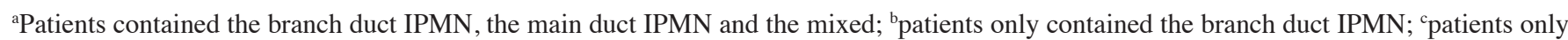
contained the main duct IPMN. CEA, carcinoembryonic antigen; CA19-9, carbohydrate antigen 19-9; IPMN, intraductal papillary mucinous neoplasm; NA, not available; QUADAS, quality assessment of diagnostic accuracy studies.

Table II. Predefined subgroup analysis of indices (with 95\% CIs) and subsequent meta-regression on DOR for CA19-9.

\begin{tabular}{|c|c|c|c|c|c|c|}
\hline Subgroup & Studies, $\mathrm{n}$ & Sensitivity $(95 \% \mathrm{CI})$ & Specificity $(95 \%$ CI) & DOR $(95 \% \mathrm{CI})$ & P-value & $\mathrm{I}^{2, \%}$ \\
\hline CA19-9 (all studies) & 15 & & & & & \\
\hline \multicolumn{7}{|l|}{ Study location } \\
\hline Asia & 14 & $0.42(0.38-0.47)$ & $0.85(0.83-0.88)$ & $3.15(1.84-5.42)$ & 0.0003 & 65.7 \\
\hline USA & 1 & NA & NA & NA & NA & NA \\
\hline \multicolumn{7}{|l|}{ QUADAS score } \\
\hline$X \leq 12$ & 5 & $0.42(0.38-0.47)$ & $0.88(0.84-0.92)$ & $4.80(1.19-19.4)$ & 0.0001 & 83.1 \\
\hline$X>12$ & 10 & $0.43(0.38-0.48)$ & $0.84(0.81-0.87)$ & $3.08(1.83-5.16)$ & 0.0448 & 47.8 \\
\hline \multicolumn{7}{|l|}{ Type of IPMN } \\
\hline All ${ }^{\mathrm{a}}$ & 9 & $0.50(0.45-0.55)$ & $0.83(0.79-0.86)$ & $4.21(1.85-9.59)$ & NA & 78.4 \\
\hline Branch $^{b}$ & 5 & $0.50(0.45-0.55)$ & $0.83(0.79-0.86)$ & $4.21(1.85-9.59)$ & NA & 78.4 \\
\hline Main $^{c}$ & 1 & NA & NA & NA & NA & NA \\
\hline
\end{tabular}

aPatients contained the branch duct IPMN, the main duct IPMN and the mixed; batients only contained the branch duct IPMN; ${ }^{\mathrm{p} p a t i e n t s}$ only contained the main duct IPMN. CI, confidence interval; DOR, diagnostic odds ratio; CA19-9, carbohydrate antigen 19-9; $\mathrm{I}^{2}$, inconsistency value; NA, not available; QUADAS, quality assessment of diagnostic accuracy studies; IPMN, intraductal papillary mucinous neoplasm.

sensitivity and specificity, indicating the absence of a threshold effect.

Several potential sources of heterogeneity were pre-identified: Study location, QUADAS score and type of IPMN. Differences in diagnostic accuracy were noted upon subgroup analysis (Table II). These differences were subtle and contributed to the heterogeneity. Study quality was the main cause of heterogeneity. Meta-regression did not identify significant differences in diagnostic accuracy among the subgroups.
Serum CA19-9 for invasive IPMN. A total of 9 studies were included (9,20-22,24,28-30,32). Pooled sensitivity was 52\% (95\% CI, 46-58\%), specificity was 88\% (95\% CI, 85-90\%), PLR was 3.78 (95\% CI, 2.83-5.05), NLR was 0.60 (95\% CI, 0.48-0.76), DOR was 6.33 (95\% CI, 3.89-10.30) and $\mathrm{I}^{2}=38.2 \%$. Parameters were homogenous and there was a significant correlation between sensitivity and specificity, indicating a threshold effect; therefore, a random-effects method was used. Forest plots of all the indices of diagnostic accuracy are shown in Fig. 8. Results 
A

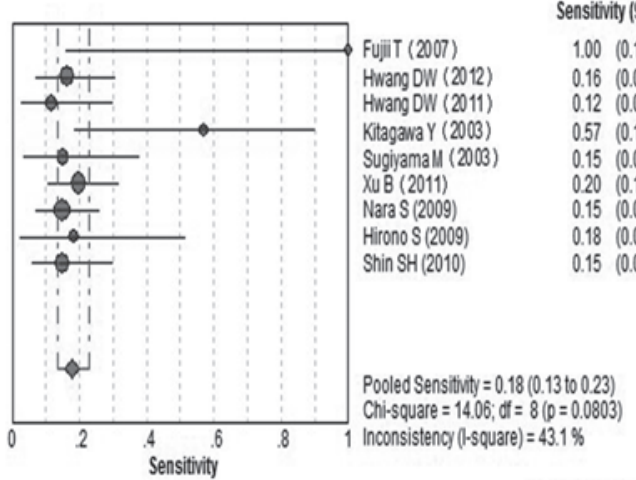

C

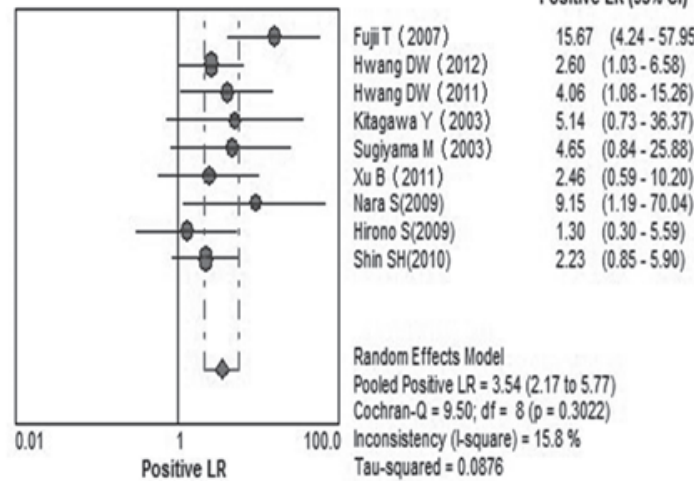

Sensitivity (95\% Cl)

$1.00(0.16-1.00)$

$0.16(0.07 \cdot 0.31)$

$0.57(0.18 \cdot 0.00)$

$0.15(0.03 \cdot 0.38)$

$0.20(0.11 \cdot 0.32)$

$0.15(0.07 \cdot 0.26)$

$0.18(0.02 \cdot 0.52$

$0.15(0.06 \cdot 0.30)$

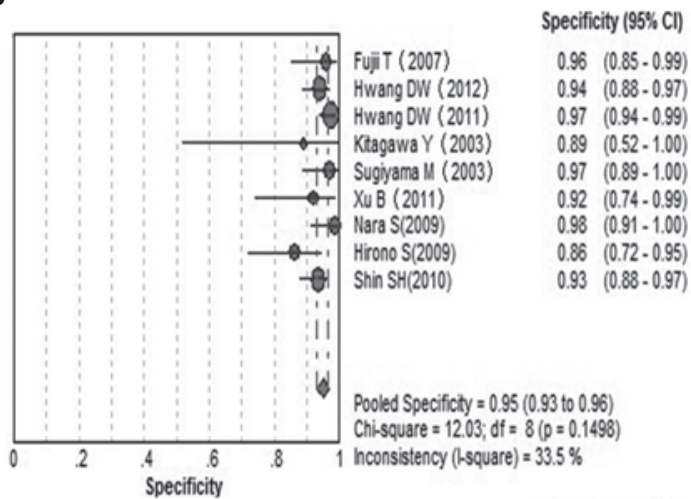

D

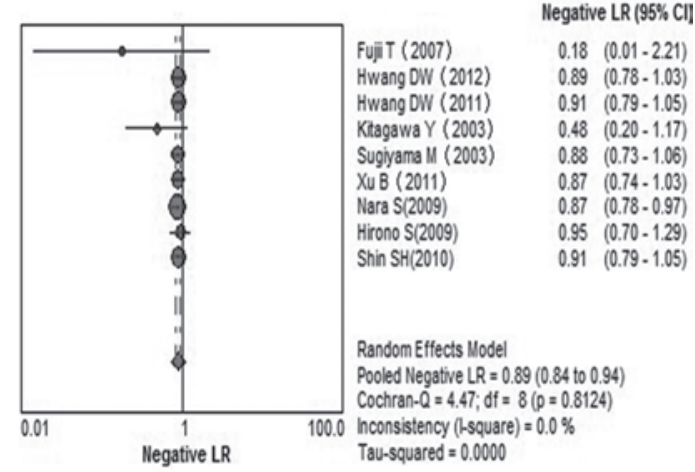

Figure 4. Forest plots of serum carcinoembryonic antigen for predicting malignant intraductal papillary mucinous neoplasms. (A) Sensitivity; (B) specificity; (C) positive-likelihood ratio (LR); and (D) negative-LR. CI, confidence interval.

were plotted as a symmetrical sROC curve (Fig. 9). The AUC was 0.78 (SE, 0.03).

\section{Discussion}

The natural history and prognosis of patients with IPMN is unknown and molecular genetic events surrounding the development of these neoplasms are under investigation. Controversy exists regarding surgical treatment (22). The correct differential diagnosis between benign and malignant IPMN is crucial for deciding on appropriate management. With small IPMN, patients with questionable malignancy on cross-sectional imaging or those with multiple co-morbidities, and raised levels of tumor markers (CA19-9 $>37 \mathrm{U} / \mathrm{ml}$ and/or CEA $\geq 5 \mu \mathrm{g} / \mathrm{ml}$ ), may facilitate the decision to proceed with surgical resection instead of conservative management. A useful marker would guide preoperative decision-making, postoperative follow-up and therapy (22). The CEA and CA19-9 tumor markers have been investigated extensively in terms of the diagnosis and prognosis of ductal adenocarcinoma (16,33-35). The present meta-analysis showed serum CEA and CA19-9 to be moderate predictors of malignancy and invasiveness.

CEA is a $180-\mathrm{kDa}$ cell-surface glycoprotein that is increased in $>60 \%$ of patients with pancreatic ductal adenocarcinoma (36). The pooled sensitivities of CEA for malignant and invasive IPMN were all 18\%, indicating an FN diagnosis, and is not useful for screening high-risk populations. Pooled specificities were 93 and $95 \%$, respectively, indicating the prediction of IPMN malignancy. The PLR of 2.83 and 3.54

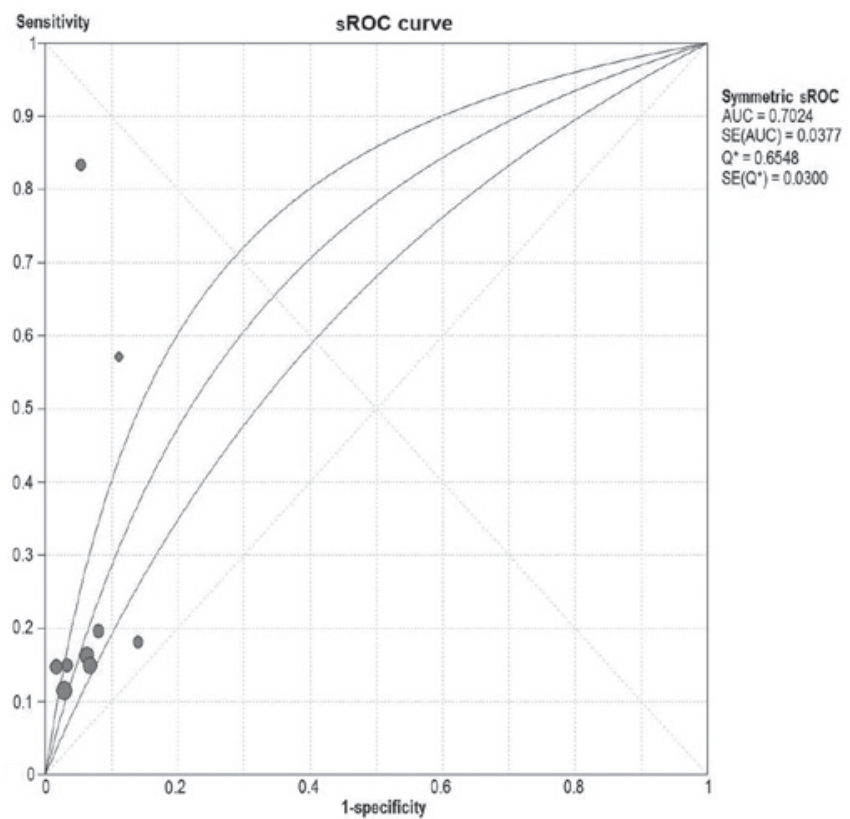

Figure 5. sROC curve of serum carcinoembryonic antigen for predicting malignant intraductal papillary mucinous neoplasms. sROC, summary receiver operating characteristic; AUC, area under the sROC curve; SE, standard error.

suggested that a CEA level $>5 \mathrm{ng} / \mathrm{ml}$ is a good predictor of malignancy and invasiveness, albeit not optimal. While a single binary threshold facilitates clinical decision-making, 
A

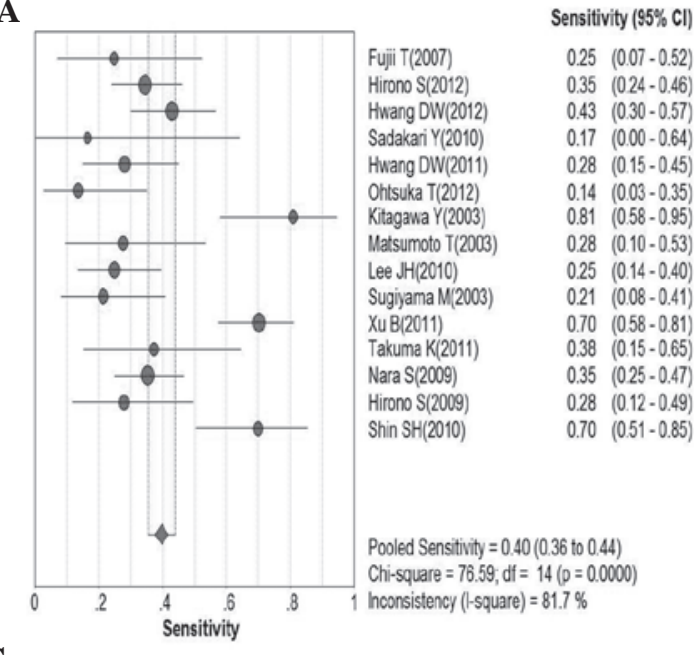

C

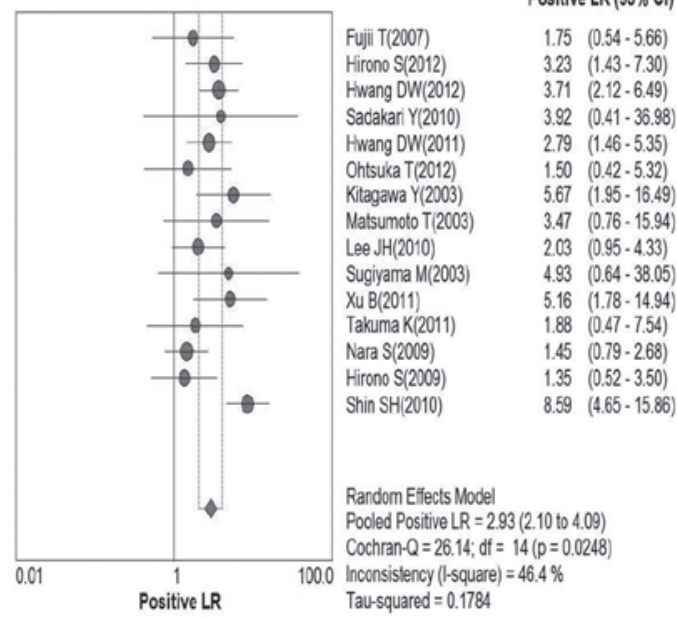

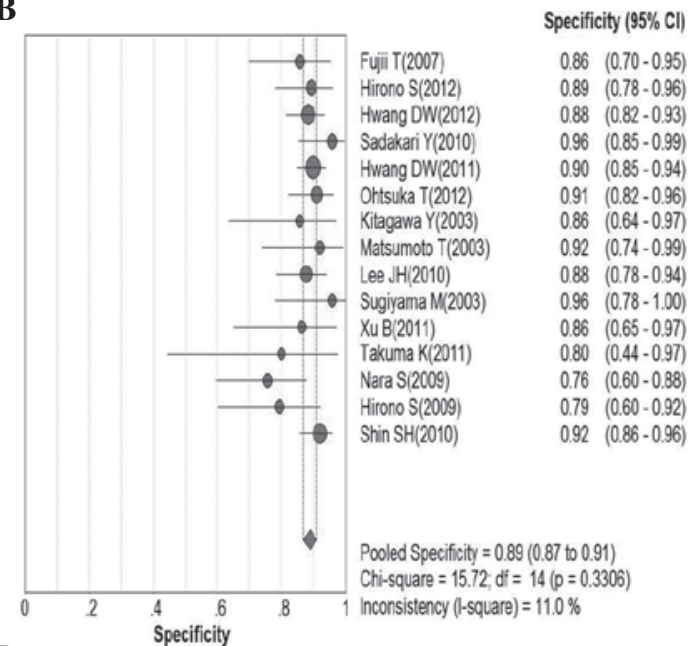

D

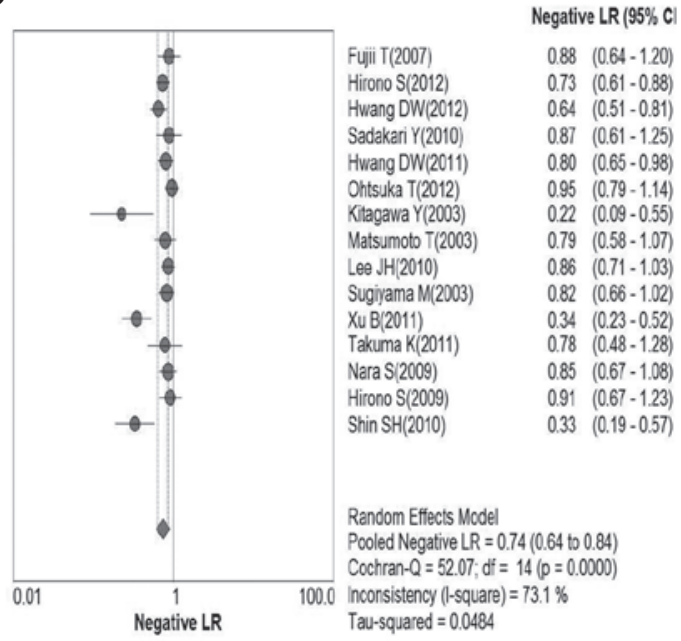

Figure 6. Forest plots of serum carbohydrate antigen 19-9 for predicting malignant intraductal papillary mucinous neoplasms. (A) Sensitivity; (B) specificity; (C) positive-likelihood ratio (LR); and (D) negative-LR. CI, confidence interval.

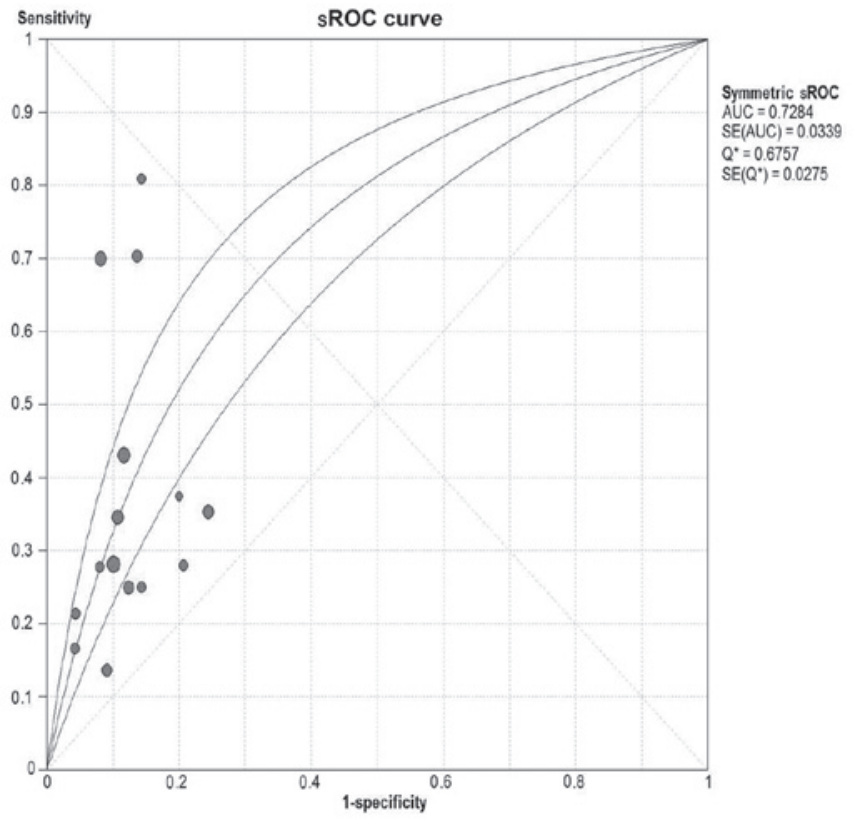

Figure 7. sROC curve of serum carbohydrate antigen 19-9 for predicting malignant intraductal papillary mucinous neoplasms. sROC, summary receiver operating characteristic; AUC, area under the sROC curve; SE, standard error. considering CEA as a spectrum may be more appropriate. Arbitrarily raising the threshold would make CEA superior for predicting malignancy, but a significant proportion of cases would not be diagnosed. CEA is released from the periphery of the cancer cell membrane and subsequently enters the systemic circulation. Analysis of cyst fluid is useful, but in certain studies pooled estimates of CEA in malignant cysts had a poor predictive value (14,37-41). Therefore, the utility of CEA levels to differentiate benign from malignant IPMN and distinguishing non-invasive from invasive IPMN were limited. The present findings indicated that clinical decisions, particularly those regarding surgery, should not be based solely on an elevated serum CEA level.

CA19-9, a tumor-associated glycoprotein, increases in $85 \%$ of patients with pancreatic ductal adenocarcinoma (42). Compared to serum CEA, serum CA19-9 is an improved predictor of malignancy. The pooled sensitivity and specificity were 44 and $85 \%$, respectively; the PLR was 2.36 and the NLR was 0.73 . There were several potential sources of heterogeneity; therefore, a subgroup analysis was performed and random-effects models were used. Subtle differences in diagnostic accuracy were noted, although these could not account for the heterogeneity. These findings strengthen the results of serum CA19-9 in the study. 
A

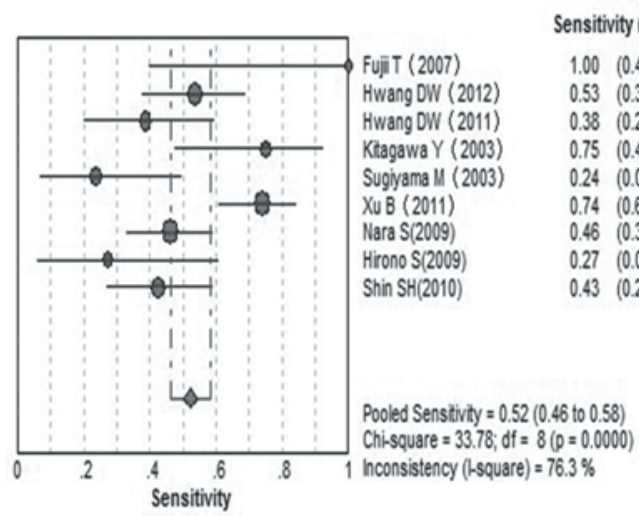

C

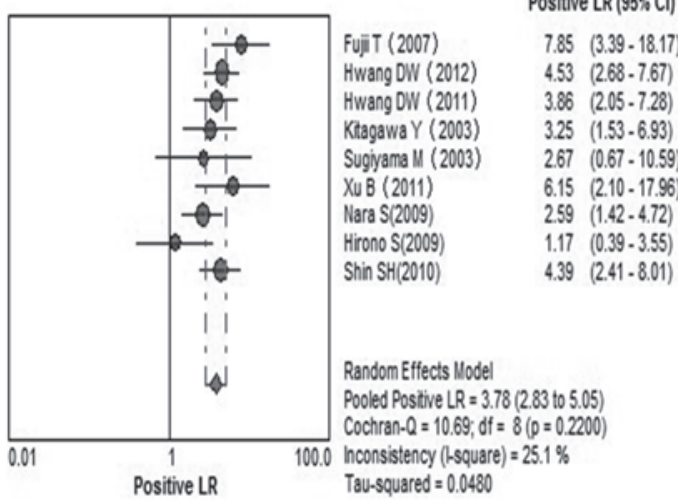

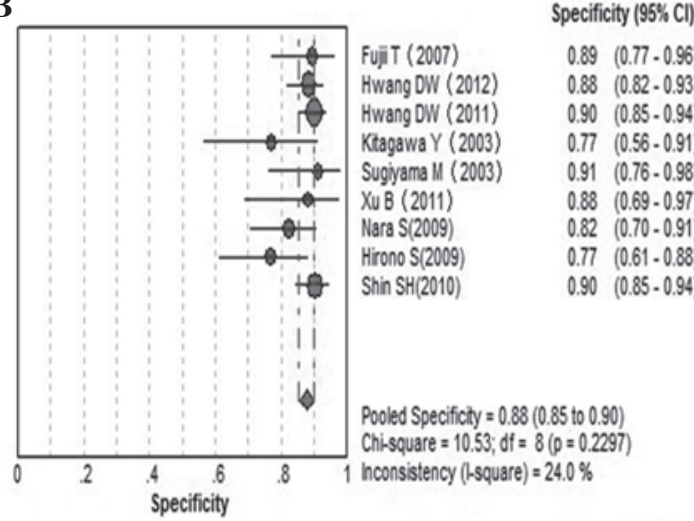

D

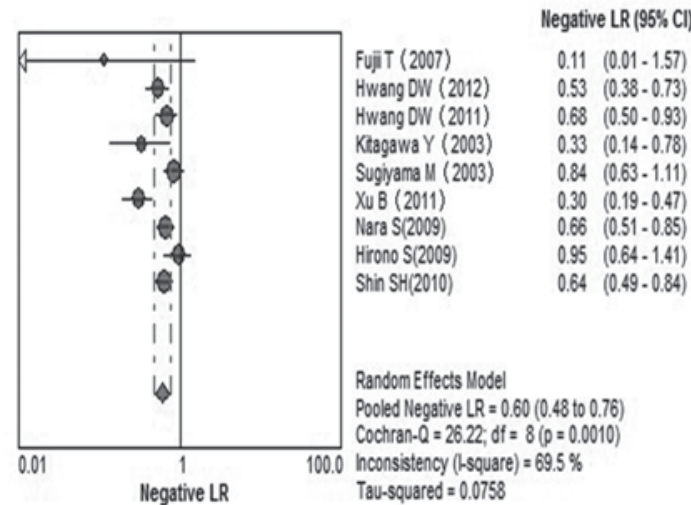

Figure 8. Forest plots of serum carbohydrate antigen 19-9 for predicting malignant intraductal papillary mucinous neoplasms. (A) Sensitivity; (B) specificity; (C) positive-likelihood ratio (LR); and (D) negative LR. CI, confidence interval.

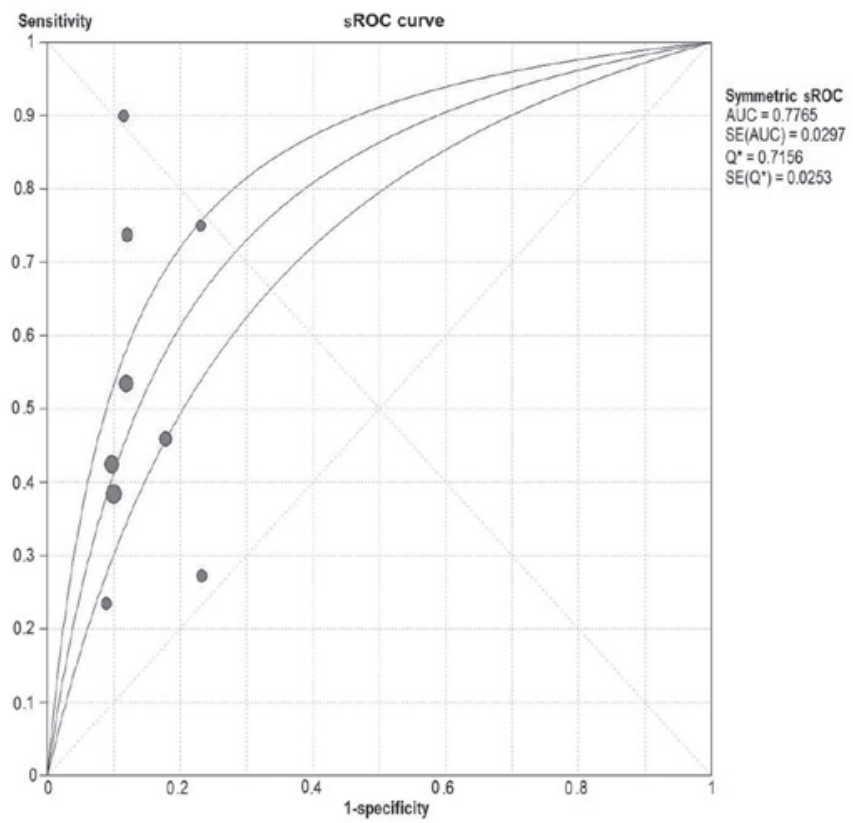

Figure 9. sROC curve of serum carbohydrate antigen 19-9 for predicting malignant intraductal papillary mucinous neoplasms. sROC, summary receiver operating characteristic; AUC, area under the sROC curve; SE, standard error.

In addition to prediction of malignancy, increased levels of CA19-9 in the present study correlated significantly with invasive IPMN. Consequently, limited resections, such as enucleation, should not be undertaken in patients with positive-tumor markers. Lymphadenectomy should be performed as part of an appropriate oncological resection.

As with any meta-analysis, the present study had limitations. There was significant heterogeneity among the studies. The study attempted to address this by employing pre-specified subgroup analysis and meta-regression, but significant heterogeneity persisted. Heterogeneity could be due to minor variations in patient populations, methods of sampling, techniques used to assay samples and the proportion of patients with malignant disease. Studies with negative results were likely unpublished, leading to publication bias. To the best of our knowledge, validation of the methods used to assay serum CEA and CA19-9 levels has not been reported.

The pooled sensitivity and specificity of CEA was inadequate to warrant their use as a diagnostic test or to replace conventional diagnostic imaging. The markers may serve as complementary tools during preoperative staging investigations of IPMN to distinguish benign and malignant tumors. Serum CA19-9 is a useful non-invasive preoperative tool for differentiating between invasive and benign IPMN and should be taken into account in the decision to perform surgery.

\section{Acknowledgements}

The present study was supported by grants from the National Natural Science Foundation of China (no. 81172315/H1617), the Research Special Fund for Public Welfare Industry of Health and the Translational Research of Early Diagnosis and 
Comprehensive Treatment in Pancreatic Cancer (no. 201202007) and the Trends and Prevention Strategies of Liver, Gallbladder, Pancreas Disease (no. 2012-XY-12-4).

\section{References}

1. Kloppel G, Solcia E, Longnecker DS, Capella C and Sobin LH (eds): Histological Typing of Tumours of the Exocrine Pancreas 2nd edition. Springer-Verlag, Berlin-Heidelberg, pp1-61, 1996.

2. Hamilton SR and Aaltonen LA (eds): Pathology and Genetics of Tumours of the Digestive System. IARC Press, Lyon, pp237-241, 2000.

3. Serikawa M, Sasaki T, Fujimoto Y, Kuwahara K and Chayama K: Management of intraductal papillary-mucinous neoplasm of the pancreas: treatment strategy based on morphologic classification. J Clin Gastroenterol 40: 856-862, 2006.

4. Yamaguchi K, Nakamura M, Shirahane K, et al: Pancreatic juice cytology in IPMN of the pancreas. Pancreatology 5: 416-421, 2005.

5. Tanaka M, Kobayashi K, Mizumoto K and Yamaguchi K: Clinical aspects of intraductal papillary mucinous neoplasm of the pancreas. J Gastroenterol 40: 669-675, 2005

6. Shimizu Y, Kanemitsu Y, Sano T, Senda Y, Mizuno N and Yamao K: A nomogram for predicting the probability of carcinoma in patients with intraductal papillary-mucinous neoplasm. World J Surg 34: 2932-2938, 2010.

7. Kimura W, Sasahira N, Yoshikawa T, Muto T and Makuuchi M: Duct-ectatic type of mucin producing tumor of the pancreas - new concept of pancreatic neoplasia. Hepatogastroenterology 43 : 692-709, 1996.

8. Sai JK, Suyama M, Kubokawa Y, et al: Management of branch duct-type intraductal papillary mucinous tumor of the pancreas based on magnetic resonance imaging. Abdom Imaging 28 694-699, 2003

9. Sugiyama M, Izumisato Y, Abe N, Masaki T, Mori T and Atomi Y: Predictive factors for malignancy in intraductal papillary-mucinous tumours of the pancreas. Br J Surg 90: 1244-1249, 2003.

10. Schmidt CM, White PB, Waters JA, et al: Intraductal papillary mucinous neoplasms: predictors of malignant and invasive pathology. Ann Surg 246: 644-654, 2007.

11. Maire F, Voitot H, Aubert A, et al: Intraductal papillary mucinous neoplasms of the pancreas: performance of pancreatic fluid analysis for positive diagnosis and the prediction of malignancy. Am J Gastroenterol 103: 2871-2877, 2008.

12. Fritz S, Hackert T, Hinz U, Hartwig W, Buchler MW and Werner J: Role of serum carbohydrate antigen 19-9 and carcinoembryonic antigen in distinguishing between benign and invasive intraductal papillary mucinous neoplasm of the pancreas. Br J Surg 98: 104-110, 2011.

13. Hartwig W, Schneider L, Diener MK, Bergmann F, Buchler MW and Werner J: Preoperative tissue diagnosis for tumours of the pancreas. Br J Surg 96: 5-20, 2009.

14. Pais SA, Attasaranya S, Leblanc JK, Sherman S, Schmidt CM and DeWitt J: Role of endoscopic ultrasound in the diagnosis of intraductal papillary mucinous neoplasms: correlation with surgical histopathology. Clin Gastroenterol Hepatol 5: 489-495, 2007.

15. Ferrone CR, Finkelstein DM, Thayer SP, Muzikansky A, Fernandez-delCastillo C and Warshaw AL: Perioperative CA19-9 levels can predict stage and survival in patients with resectable pancreatic adenocarcinoma. J Clin Oncol 24: 2897-2902, 2006.

16. Sandblom G, Granroth S and Rasmussen IC: TPS, CA 19-9, VEGF-A, and CEA as diagnostic and prognostic factors in patients with mass lesions in the pancreatic head. Ups J Med Sci 113: 57-64, 2008.

17. Smith RA, Bosonnet L, Ghaneh P, et al: Preoperative CA19-9 levels and lymph node ratio are independent predictors of survival in patients with resected pancreatic ductal adenocarcinoma. Dig Surg 25: 226-232, 2008.

18. Zamora J, Abraira V, Muriel A, Khan K and Coomarasamy A: Meta-DiSc: a software for meta-analysis of test accuracy data. BMC Med Res Methodol 6: 31, 2006.

19. Ohtsuka T, Kono H, Nagayoshi Y, et al: An increase in the number of predictive factors augments the likelihood of malignancy in branch duct intraductal papillary mucinous neoplasm of the pancreas. Surgery 151: 76-83, 2012

20. Hwang DW, Jang JY, Lee SE, Lim CS, Lee KU and Kim SW: Clinicopathologic analysis of surgically proven intraductal papillary mucinous neoplasms of the pancreas in SNUH: a 15-year experience at a single academic institution. Langenbecks Arch Surg 397: 93-102, 2012.
21. Hirono S, Tani M, Kawai M, et al: The carcinoembryonic antigen level in pancreatic juice and mural nodule size are predictors of malignancy for branch duct type intraductal papillary mucinous neoplasms of the pancreas. Ann Surg 255: 517-522, 2012.

22. Xu B, Zheng WY, Jin DY, Ding WX, Lou WH and Ramsohok L: Predictive value of serum carbohydrate antigen 19-9 in malignant intraductal papillary mucinous neoplasms. World J Surg 35: 1103-1109, 2011

23. Takuma K, Kamisawa T, Anjiki H, et al: Predictors of malignancy and natural history of main-duct intraductal papillary mucinous neoplasms of the pancreas. Pancreas 40: 371-375, 2011.

24. Hwang DW, Jang JY, Lim CS, et al: Determination of malignant and invasive predictors in branch duct type intraductal papillary mucinous neoplasms of the pancreas: a suggested scoring formula. J Korean Med Sci 26: 740-746, 2011.

25. Shin SH, Han DJ, Park KT, Kim YH, Park JB and Kim SC: Validating a simple scoring system to predict malignancy and invasiveness of intraductal papillary mucinous neoplasms of the pancreas. World J Surg 34: 776-783, 2010.

26. Sadakari Y, Ienaga J, Kobayashi K, et al: Cyst size indicates malignant transformation in branch duct intraductal papillary mucinous neoplasm of the pancreas without mural nodules. Pancreas 39: 232-236, 2010.

27. Lee JH, Lee KT, Park J, et al: Predictive factors associated with malignancy of intraductal papillary mucinous pancreatic neoplasms. World J Gastroenterol 16: 5353-5358, 2010.

28. Nara S, Onaya H, Hiraoka N, et al: Preoperative evaluation of invasive and noninvasive intraductal papillary-mucinous neoplasms of the pancreas: clinical, radiological, and pathological analysis of 123 cases. Pancreas 38: 8-16, 2009.

29. Hirono S, Tani M, Kawai M, et al: Treatment strategy for intraductal papillary mucinous neoplasm of the pancreas based on malignant predictive factors. Arch Surg 144: 345-350, 2009.

30. Fujii T, Ishikawa T, Kanazumi N, et al: Analysis of clinicopathological features and predictors of malignancy in intraductal papillary mucinous neoplasms of the pancreas. Hepatogastroenterology 54: 272-277, 2007.

31. Matsumoto T, Aramaki M, Yada K, et al: Optimal management of the branch duct type intraductal papillary mucinous neoplasms of the pancreas. J Clin Gastroenterol 36: 261-265, 2003.

32. Kitagawa Y, Unger TA, Taylor S, Kozarek RA and Traverso LW: Mucus is a predictor of better prognosis and survival in patients with intraductal papillary mucinous tumor of the pancreas. J Gastrointest Surg 7: 12-19, 2003.

33. Katz MH, Varadhachary GR, Fleming JB, et al: Serum CA 19-9 as a marker of resectability and survival in patients with potentially resectable pancreatic cancer treated with neoadjuvant chemoradiation. Ann Surg Oncol 17: 1794-1801, 2010.

34. Sedlack R, Affi A, Vazquez-Sequeiros E, Norton ID, Clain JE and Wiersema MJ: Utility of EUS in the evaluation of cystic pancreatic lesions. Gastrointest Endosc 56: 543-547, 2002.

35. Tatsuta M, Iishi H, Ichii M, et al: Values of carcinoembryonic antigen, elastase 1 , and carbohydrate antigen determinant in aspirated pancreatic cystic fluid in the diagnosis of cysts of the pancreas. Cancer 57: 1836-1839, 1986.

36. Satake K, Kanazawa G, Kho I, Chung Y and Umeyama K: Evaluation of serum pancreatic enzymes, carbohydrate antigen 19-9, and carcinoembryonic antigen in various pancreatic diseases. Am J Gastroenterol 80: 630-636, 1985.

37. Ngamruengphong S, Bartel MJ and Raimondo M: Cyst carcinoembryonic antigen in differentiating pancreatic cysts: a meta-analysis. Dig Liver Dis 45: 920-926, 2013.

38. Othman MO, Patel M, Dabizzi E, et al: Carcino Embryonic Antigen and long-term follow-up of mucinous pancreatic cysts including intraductal papillary mucinous neoplasm. Dig Liver Dis 44: 844-848, 2012.

39. Correa-Gallego C, Warshaw AL and Fernandez-del Castillo C: Fluid CEA in IPMNs: A useful test or the flip of a coin? Am J Gastroenterol 104: 796-797, 2009.

40. Park WG, Mascarenhas R, Palaez-Luna M, et al: Diagnostic performance of cyst fluid carcinoembryonic antigen and amylase in histologically confirmed pancreatic cysts. Pancreas 40: 42-45, 2011.

41. Nagula S, Kennedy T, Schattner MA, et al: Evaluation of cyst fluid CEA analysis in the diagnosis of mucinous cysts of the pancreas. J Gastrointest Surg 14: 1997-2003, 2010.

42. Safi F, Schlosser W, Falkenreck S and Beger HG: Prognostic value of CA 19-9 serum course in pancreatic cancer. Hepatogastroenterology 45: 253-259, 1998. 